\title{
Video Article \\ Enhanced Rabies Surveillance Using a Direct Rapid Immunohistochemical Test
}

\author{
Erin M. Patrick ${ }^{1}$, Brian M. Bjorklund ${ }^{2}$, Jordona D. Kirby ${ }^{3}$, Kathleen M. Nelson ${ }^{4}$, Richard B. Chipman ${ }^{4}$, Charles E. Rupprecht ${ }^{5}$ \\ ${ }^{1}$ Animal and Plant Health Inspection Service (APHIS), Wildlife Services, Knoxville, TN, United States Department of Agriculture (USDA) \\ ${ }^{2}$ Animal and Plant Health Inspection Service (APHIS), Wildlife Services, Sutton, MA, United States Department of Agriculture (USDA) \\ ${ }^{3}$ Animal and Plant Health Inspection Service (APHIS), Wildlife Services, Milton, FL, United States Department of Agriculture (USDA) \\ ${ }^{4}$ Animal and Plant Health Inspection Service (APHIS), Wildlife Services, Concord, NH, United States Department of Agriculture (USDA) \\ ${ }^{5}$ Lyssa LLC
}

Correspondence to: Erin M. Patrick at erin.patrick@usda.gov

URL: https://www.jove.com/video/59416

DOI: doi: $10.3791 / 59416$

Keywords: Immunology and Infection, Issue 146, diagnosis, direct rapid immunohistochemical test, fluorescent antibody test, lyssavirus, rabies, zoonosis

Date Published: 4/30/2019

Citation: Patrick, E.M., Bjorklund, B.M., Kirby, J.D., Nelson, K.M., Chipman, R.B., Rupprecht, C.E. Enhanced Rabies Surveillance Using a Direct Rapid Immunohistochemical Test. J. Vis. Exp. (146), e59416, doi:10.3791/59416 (2019).

\section{Abstract}

Laboratory-based surveillance is integral for rabies prevention, control and management efforts. While the DFA is the gold standard for rabies diagnosis, there is a need to validate additional diagnostic techniques to improve rabies surveillance, particularly in developing countries. Here, we present a standard protocol for the DRIT as an alternative, laboratory or field-based testing option that uses light microscopy as compared to the DFA. Touch impressions of brain tissue collected from suspect animals are fixed in $10 \%$ buffered formalin. The DRIT uses rabies virusspecific monoclonal or polyclonal antibodies (conjugated to biotin), a streptavidin-peroxidase enzyme, and a chromogen reporter (such as acetyl 3-amino-9-ethylcarbazole) to detect viral inclusions within infected tissue. In approximately $1 \mathrm{~h}$, a brain tissue sample can be tested and interpreted by the DRIT. Evaluation of suspect animal brains tested from a variety of species in North America, Asia, Africa, and Europe have illustrated high sensitivity and specificity by the DRIT approaching $100 \%$ with results compared to DFA. Since 2005 , the United States Department of Agriculture's Wildlife Services (USDA WS) program has conducted large-scale enhanced rabies surveillance efforts using the DRIT to test $>94,000$ samples collected from wildlife in strategic rabies management areas. The DRIT provides a powerful, economical tool for rabies diagnosis that can be used by laboratorians and field biologists to improve current rabies surveillance, prevention and control programs globally.

\section{Video Link}

The video component of this article can be found at https://www.jove.com/video/59416/

\section{Introduction}

While the DFA is the most widely used test for routine rabies diagnosis ${ }^{1}$, the cost of purchasing and maintaining a fluorescent microscope can be limiting to developing nations $\mathrm{s}^{2,3,4}$ and for broad, large-scale enhanced rabies surveillance programs $\mathrm{s}^{3,4}$. In addition, the DFA requires the ability to refrigerate samples during fixation and to incubate samples above ambient temperature during the antibody-antigen reactions, which can be a significant hurdle in countries without suitable infrastructure. Due in part to the limitations associated with modern DFA testing, the global impact of rabies has long been underestimated ${ }^{5}$.

In this regard, there is a need to validate additional diagnostic techniques to improve rabies surveillance globally, particularly in developing countries. The DRIT protocol presented here offers a laboratory or field-based testing alternative to the DFA that uses light microscopy and does not require refrigeration or laboratory incubation during the test ${ }^{6}$. The DRIT and DFA are similar in that both techniques use touch impressions of brain samples collected from potentially rabid animals. However, the first step of the DRIT uses formalin as an historical fixative for samples, which inactivates rabies virus. This provides a substantial biosafety improvement over the acetone used to fix samples in the DFA protocol ${ }^{3}$, which is important not only in the laboratory but perhaps even more so in field-based or decentralized lab environments. To date, the DRIT shows sensitivity and specificity equal to the DFA $\mathrm{DF}^{2,7,8,9}$.

Since 2005, the USDA WS program has conducted large-scale enhanced rabies surveillance efforts in North America using the DRIT as part of a comprehensive wildlife rabies management program ${ }^{10}$. Enhanced rabies surveillance is used as a complement to exposure-based public health surveillance and tests primarily wildlife meso-carnivore species, including raccoons (Procyon lotor), striped skunks (Mephitis mephitis), gray foxes (Urocyon cinereoargenteus), red foxes (Vulpes vulpes), and coyotes (Canis latrans) that have not been involved in a human or domestic animal exposure. Animal carcasses used in testing were submitted to USDA WS through enhanced rabies surveillance efforts and comprised rabies vector species that are: ill or strange acting; found dead, road-killed, or a nuisance; and are not associated with a human or domestic animal exposure. The DRIT provides a rabies diagnostic test that can be employed by trained field biologists with little or no laboratory 
experience to improve rabies surveillance and reduce the financial burden and workload of public health diagnostic laboratories ${ }^{10}$. The basic DRIT training for USDA WS field employees typically takes 1.5 to 2 days, which includes approximately $4 \mathrm{~h}$ of classroom instruction covering fundamental principles of biosafety, methods for sample collection and the DRIT processes as well as $>8 \mathrm{~h}$ of laboratory time conducting the test. Training opportunities have been available to USDA WS and program cooperators through the Centers for Disease Control and Prevention (CDC), LYSSA LLC, and The Wistar Institute.

Within strategic management areas, USDA WS has tested over 94,000 wildlife samples using the DRIT and has detected more than 1,850 rabid samples that would have likely gone undetected relying only upon exposure based public health testing of suspect animals. Enhanced rabies surveillance data provided by DRIT results are critical to providing a more complete temporal and spatial representation of rabies on the landscape in support of oral rabies vaccination programs for wildlife throughout the USA ${ }^{10,11}$. Similarly, Provincial wildlife agencies in Canada have incorporated DRIT into large scale wildlife rabies surveillance efforts in tandem with their public health programs with success ${ }^{8}$. Both USDA WS and Canadian DRIT surveillance programs have used national reference laboratories to confirm rabies by DFA and to perform viral variant typing as appropriate ${ }^{8}$. Moreover, USDA WS field biologists send $10 \%$ of negative specimens for DFA confirmation to reference laboratories and since 2008, have participated in biannual DRIT proficiency testing provided by the Wisconsin State Laboratory of Hygiene, as part of standard quality assurance measures.

The goal of this method is to offer an alternative approach for rabies diagnostic testing that can be done in decentralized laboratories, in the field, or in areas without routine access to electron microscopy.

\section{Protocol}

Each person conducting rabies diagnostic testing should receive a standard pre-exposure rabies vaccination series and undergo regular serological antibody evaluation, with booster immunizations as needed. Unimmunized individuals should not enter laboratories or areas where such work is conducted. All manipulation of tissues and slides should be conducted to not aerosolize liquids or produce airborne particles. Fume hoods are not required but when feasible they can provide extra protection from odors, ectoparasites, and bone fragments. Minimum personal protective equipment, including gloves and eye protection, should be worn at all times during sample collection and testing.

\section{Brainstem Collection}

1. Collect brainstem samples either immediately upon carcass collection or ensure that carcasses are placed in freezers for storage until later testing. If animal carcasses are frozen, thaw at ambient temperature prior to brainstem collection for ease of collection. Under some circumstances, samples are collected directly from a frozen animal.

2. For animals that are freshly collected or have been thawed to ambient temperature, position the animal supine on a flat surface with the cervical spine slightly rotated towards the examiner. Palpate to identify the atlanto-occipital joint on the lateral aspect of the cervical spine.

1. For carcasses that are still completely frozen, position the animal supine as described above, if practical. Use saws, knives, bone shears or similar equipment to completely separate the head from the body.

NOTE: All equipment used that is not one-time use should be cleaned thoroughly between samples.

3. Using a scalpel blade, make an incision at the level of the atlanto-occipital joint at the ventral aspect, cutting through all layers of muscle and soft tissue, including the trachea and esophagus. Continue until approximating anterior aspect of cervical spine vertebrae. For frozen tissue, use a scalpel blade to remove any remaining layers of muscle and soft tissue to expose the foramen magnum.

4. Move the head of the animal into end-range extension until the brainstem is visible. Use a scalpel blade to remove all visible brainstem/ central nervous system (CNS) tissue for sampling. For a frozen sample, use a scalpel blade to remove as much of the frozen brainstem/CNS tissue as possible from inside the skull.

5. Place the brainstem samples in an unbreakable container (i.e., metal ointment tin, cryo-vial, etc.) and label accordingly.

6. Ensure that the brainstem samples are tested immediately after collection via the DRIT test, refrigerated $\left(4^{\circ} \mathrm{C}\right)$ for up to $24 \mathrm{~h}$ before testing, or frozen $\left(-20^{\circ} \mathrm{C}\right)$ until time of testing if testing will take place more than $24 \mathrm{~h}$ after sample collection.

\section{Preparation of Materials for DRIT}

1. Setting up of 10 slide staining dishes (Figure 1)

NOTE: Staining dishes are deep enough to allow for complete immersion of slide (diameter $96 \mathrm{~mm}$, height $72 \mathrm{~mm}$, depth $42 \mathrm{~mm}$, volume 250 $\mathrm{mL})^{6}$

1. Fill dish 1 with $10 \%$ phosphate buffered formalin. Replace formalin after 2 test runs or weekly.

2. Fill dishes 2,4 , and 5 with phosphate buffered saline with $1 \%$ tween- 80 (TPBS). Replace with fresh TPBS before each test.

3. Fill dish 3 with $3 \%$ hydrogen peroxide. Replace hydrogen peroxide before each test.

4. Fill dishes $6,8,9$, and 10 with distilled or deionized water. Replace water before each test.

5. Fill dish 7 with Gills hematoxylin formulation \#2 diluted in a 1:1 ratio in distilled water ${ }^{6}$. Replace hematoxylin after 2 test runs or weekly. NOTE: According to the original DRIT protocol developed by the $\operatorname{CDC}^{12}$, a 1:2 ratio of Gills hematoxylin to water may be used if counterstaining is too dark.

2. Preparation of amino-ethylcarbazole (AEC) stock solution

1. Using a glass pipette, place $5 \mathrm{~mL}$ of $\mathrm{N}, \mathrm{N}$-dimethylformamide in a glass container.

2. Add one $20 \mathrm{mg}$ tablet of 3-amino-9-ethylcarbazole and shake until completely dissolved. Label the jar with "AEC stock" and the date the stock was made.

NOTE: The AEC stock solution can be stored under refrigeration $\left(4^{\circ} \mathrm{C}\right)$ and used for $1-2$ months. 


\section{Preparation of AEC working dilution}

1. Add $7 \mathrm{~mL}$ of acetate buffer to a $15 \mathrm{~mL}$ centrifuge tube.

2. Using a glass pipette, add $0.5 \mathrm{~mL}$ of the $A E C$ stock solution to the centrifuge tube.

3. Add $0.075 \mathrm{~mL}$ of $3 \%$ hydrogen peroxide to the tube.

4. Filter the solution with a $10 \mathrm{~mL}$ syringe using a $0.45 \mu \mathrm{m}$ syringe filter. NOTE: The AEC working dilution should be created just prior to each DRIT test as it is only stable for $2-3 \mathrm{~h}$.

\section{Direct Rapid Immunohistochemistry Test}

1. Label glass microscope slides with a unique number for each specimen using a smear-proof, waterproof, permanent ink marker.

1. Using a scalpel blade, remove the brainstem from the container and place on paper towel. Gently blot away any excess of fluid, blood, or fur with a second paper towel to reveal just the brainstem tissue ${ }^{13}$. If needed, section the brainstem tissue to reveal a cross section.

2. Very gently touch the microscope slide to the cross section of the brainstem tissue. Touch the slide to the brainstem at several points without lateral movement to allow multiple areas of brainstem to be transferred to slide ${ }^{13}$. Ensure that only 1 or 2 layers of cells are transferred from the brain tissue to the slide with a gentle touch. No mounting agent is needed to affix the brainstem tissue to the slides. Include both a positive and negative control in each DRIT run.

2. Let the slides air-dry for approximately $5 \mathrm{~min}$ at room temperature.

3. Immerse the slides in $10 \%$ buffered formalin for 10 min (dish 1 ).

4. Remove the slides from formalin and dip-rinse in a solution of TPBS (dish 2).

5. Immerse the slides in $3 \%$ hydrogen peroxide for 10 min (dish 3 ).

6. Remove the slides from the hydrogen peroxide and dip-rinse in fresh TPBS (dish 4). After removing excess hydrogen peroxide, place the slides in fresh TPBS (dish 5) and work with one slide at a time while the other slides stay immersed in TPBS.

7. Take the slides from TPBS (dish 5) one at a time, shake off and blot excess buffer, and place on a moistened paper towel on lab bench, as the basis of a 'humidity chamber'. Using a pipette, drop enough primary anti-rabies virus antibody on each slide to cover the CNS tissue. Incubate the slides for $10 \mathrm{~min}$ in the humidity chamber (i.e., covering slides with well plates or other simple cover while they are laying on the moistened paper towel) at room temperature (Figure 2).

8. Remove the slides from the humidity chamber, shake and blot off excess conjugate, and dip-rinse the slides in TPBS (reuse the same TPBS in dish 5).

9. Working with one slide at a time, while others stay immersed in TPBS - use a pipette to drop enough streptavidin-peroxidase complex to cover the CNS tissue. Incubate in the humidity chamber for $10 \mathrm{~min}$ at room temperature.

10. Remove the slides from the humidity chamber, shake and blot off excess complex, and dip-rinse the slides in TPBS (dish 5).

11. Working with one slide at a time, shake off and blot excess buffer, while others stay immersed in TPBS - use a pipette to drop enough AEC (preparation is explained above and should be done immediately prior to use) to cover the CNS tissue. Incubate in the humidity chamber for 10 min at room temperature.

12. Dip-rinse the slides in distilled water (dish 6).

13. Place the slides in counterstain of Gills Hematoxylin (diluted 1:1 with distilled water) for 2 min (dish 7).

14. Immediately dip-rinse all slides in distilled water (dish 8). Repeat twice with fresh water each time (dishes 9 and 10).

15. Working with one slide at a time, while others stay immersed in distilled water (dish 10) - shake and blot off excess water and use a watersoluble mounting medium to affix a cover slip.

16. Use a light microscope with a $20 x$ objective to view the slides and a $40 x$ objective if closer inspection is needed.

\section{Representative Results}

Positive results from the DRIT show red intracytoplasmic viral inclusions that can vary in shape and size (Figure 3) within the cytoplasm of bluish cell bodies. The inclusions appear smooth with very bright margins and a less intensively stained central area. Intensity and antigen distribution are recorded when inclusions are detected. Intensity is graded from +4 to +1 . The positive control slide should have an intense, glaring magenta brilliance which is referred to as a +4 intensity. A slight loss of color can occur especially when sample handling has not been optimal (i.e., sample tissue has decomposed slightly) and these should be graded as +3 . Noticeably dull stain is graded as a +2 to +1 , is not considered diagnostic for rabies virus infection and is labeled as indeterminate.

Additionally, antigen distribution is graded from +4 to +1 with +4 representing antigen distribution comprised of an abundance of large and small inclusions varying in size and shape, and present in every field (or almost every field) of view within the CNS tissue touch impression. The positive control typically has a +4 antigen distribution. An antigen distribution of +3 would be assigned when there are inclusions in a variety of sizes in most but not all of the fields of view. If inclusions are found in $10 \%-50 \%$ of the microscope fields, a +2 antigen distribution is assigned. When inclusions are found in $<10 \%$ of the microscope fields, $a+1$ antigen distribution is assigned.

Most CNS tissue with rabies virus present exhibit typical viral inclusions graded as +3 or +4 intensity and antigen distribution. If results indicate $a+2$ or +1 intensity or $a+2$ or +1 antigen distribution, the sample is declared an "indeterminate" and repeat testing is warranted. If the same sample has a repeat indeterminate test result, the sample should be sent to a reference laboratory for DFA or related confirmatory testing.

A test sample using the DRIT is considered negative for rabies virus antigens after the slide containing the CNS tissue has been scanned at a magnification of 200X or greater and no typical virus inclusions are detected (Figure 4). Negative samples exhibit bluish cell bodies with little or no nonspecific staining. 


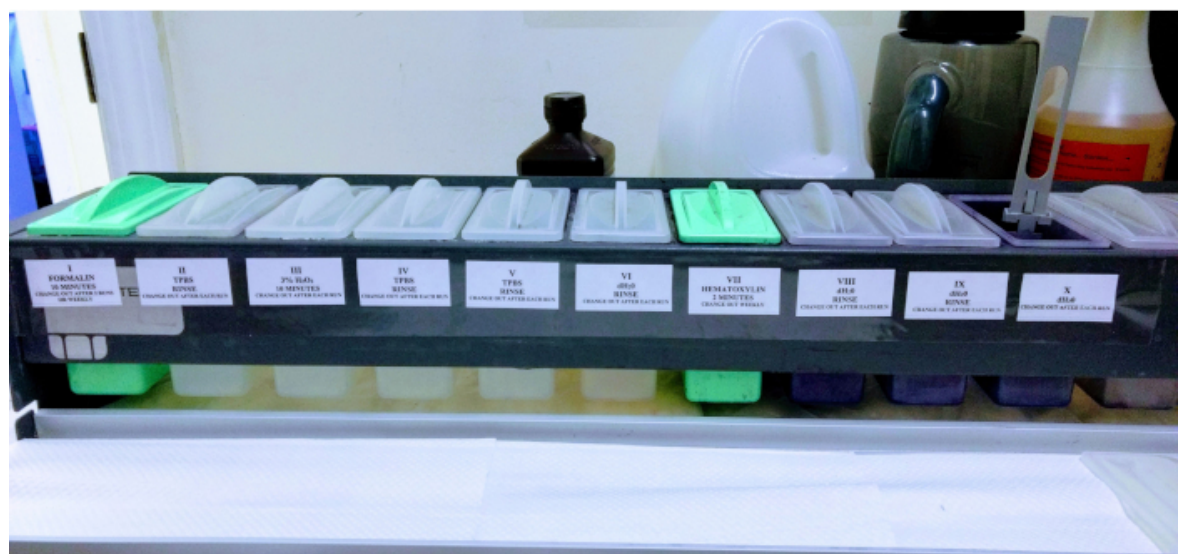

Figure 1: Setup of 10 slide staining dishes with reagents for testing. The dishes are labeled with reagent name in the order needed to follow the protocol. Please click here to view a larger version of this figure.

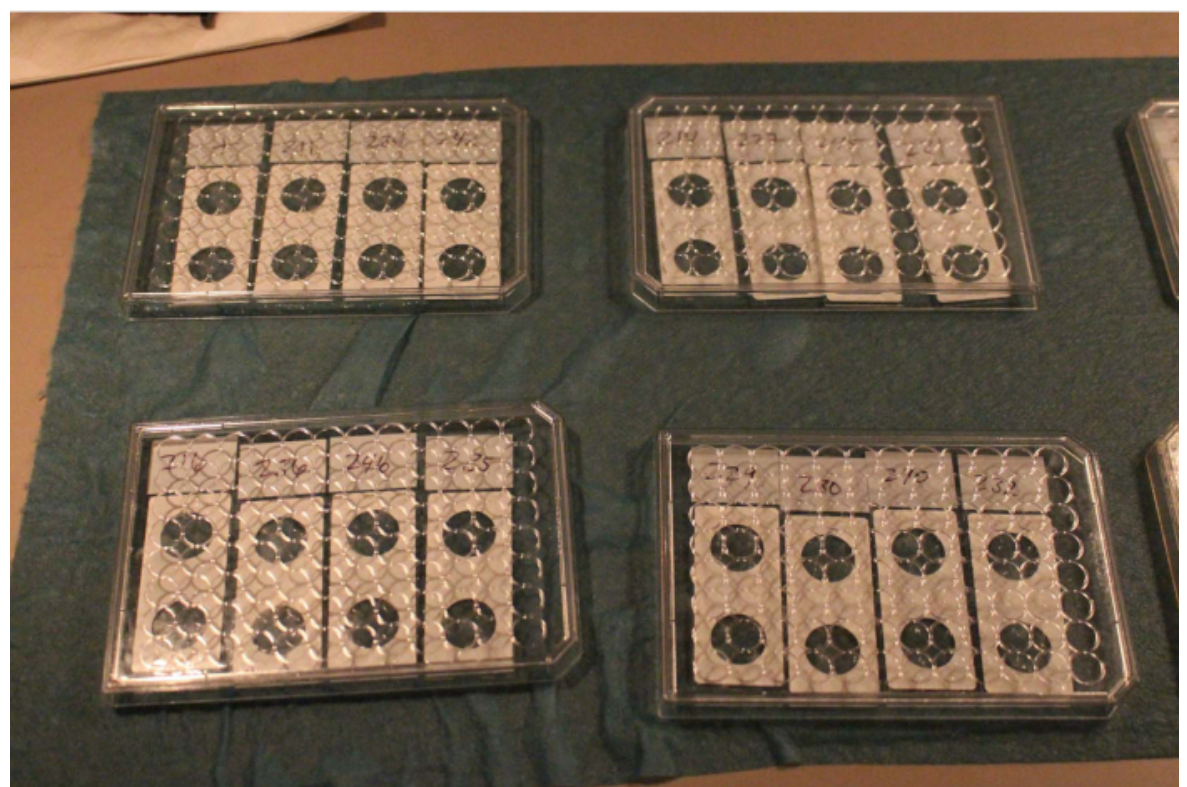

Figure 2: Simple humidity chamber created with a wet paper towel and cell culture plates. A simple humidity chamber using a wet paper towel and cell culture plates allows for field-based application. Please click here to view a larger version of this figure. 

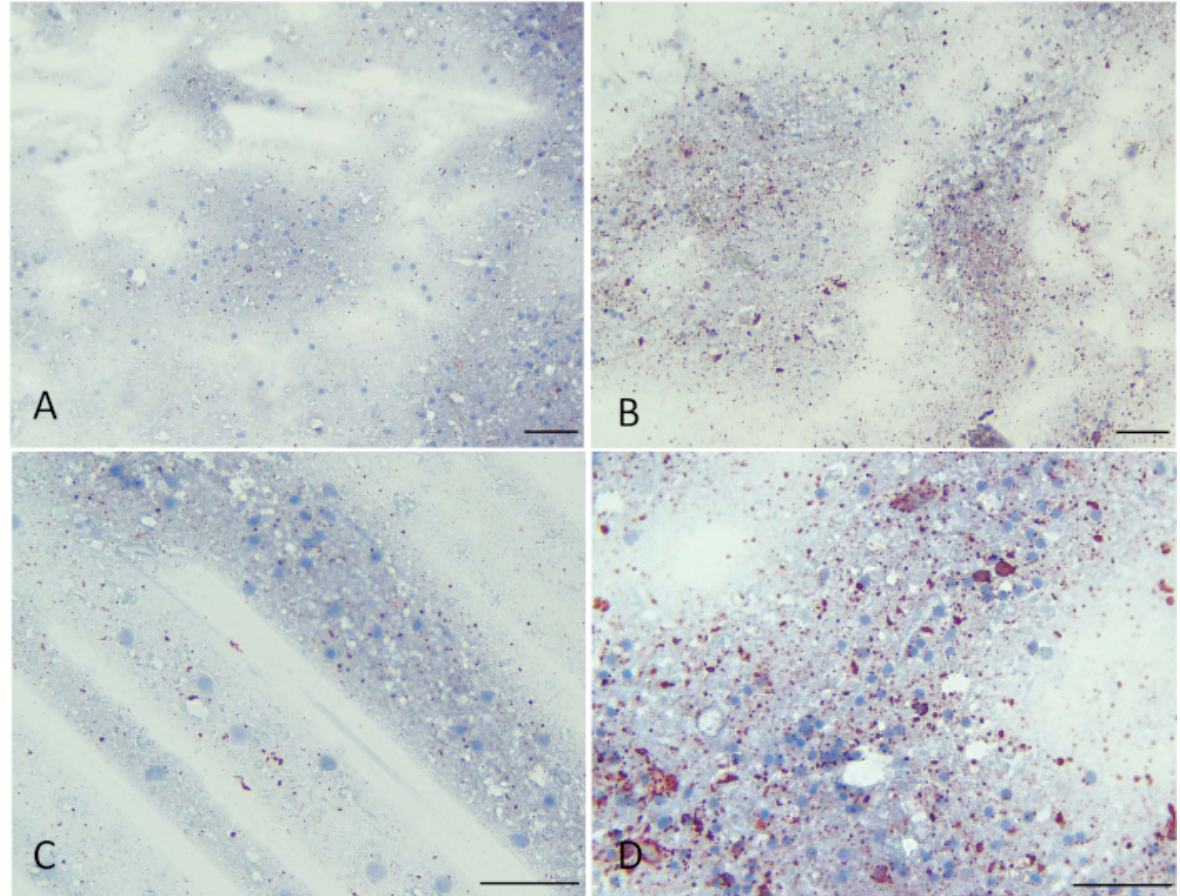

C.

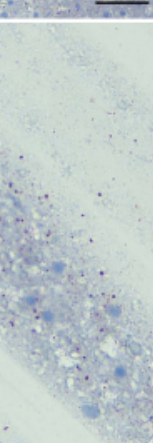

Figure 3: Representative slides of rabies positive viral inclusions with +4 intensity and +4 antigen distribution. (A and $B$ ) show positive rabies viral inclusions at 200x magnification. (C and D) show positive rabies viral inclusions at $400 x$ magnification. Scale bars $=5 \mu \mathrm{m}$. Please click here to view a larger version of this figure.
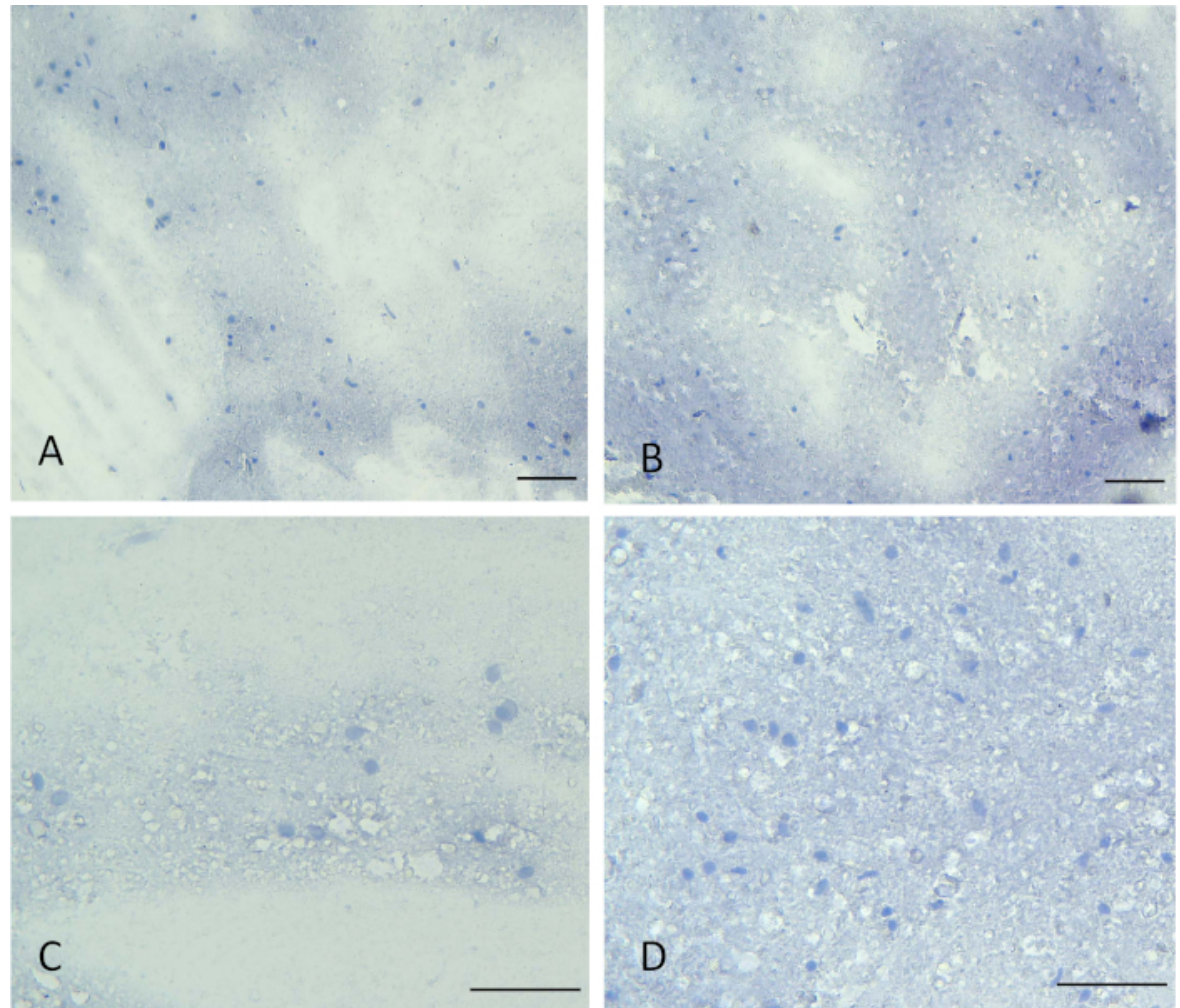

Figure 4: Representative slides of negative samples with no rabies viral inclusions. (A and B) show samples negative for rabies viral inclusions at 200x magnification. (C and D) show samples negative for rabies viral inclusions at $400 x$ magnification. Scale bars $=5 \mu \mathrm{m}$. Please click here to view a larger version of this figure. 

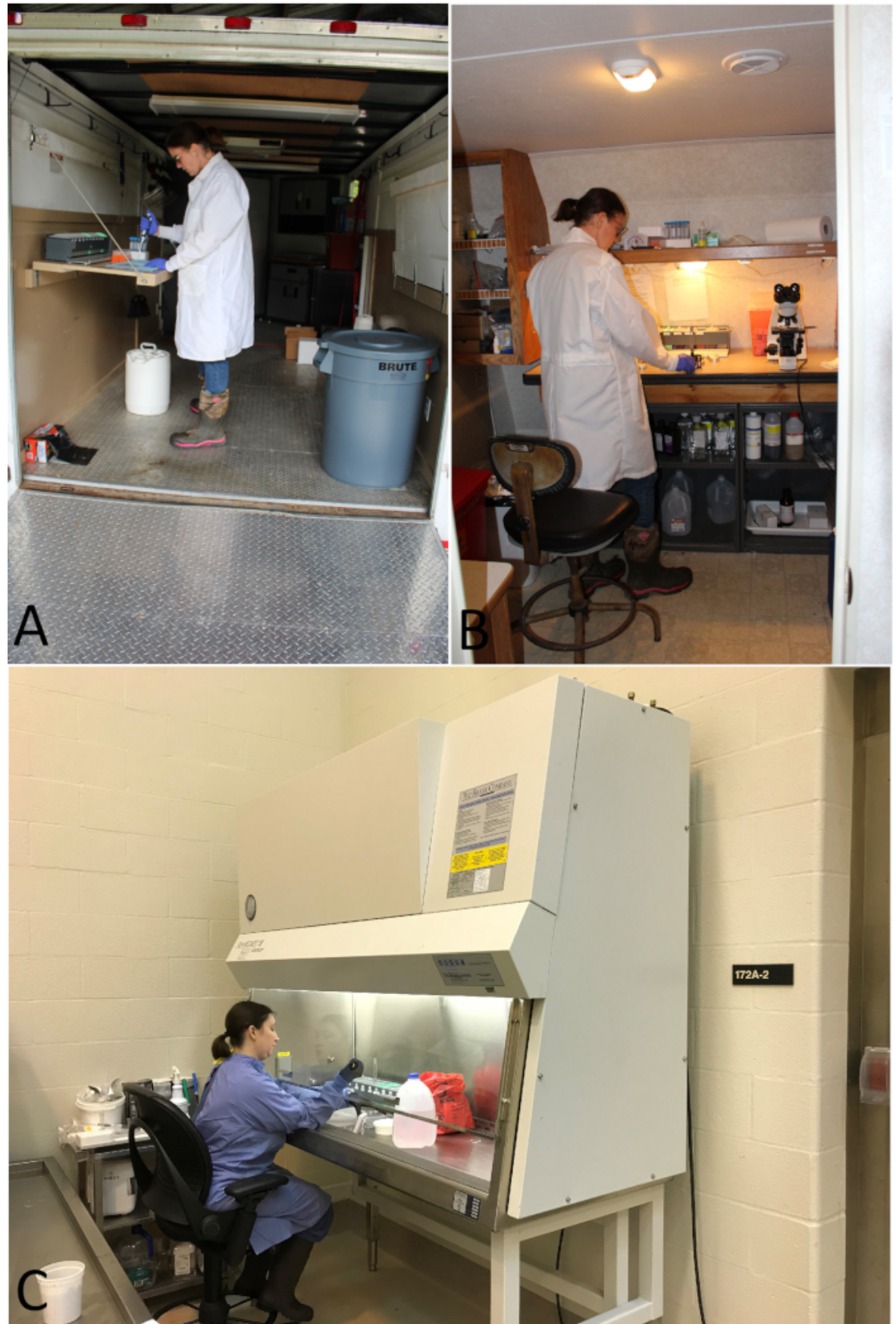

Figure 5: Representative photographs of DRIT testing facilities used by USDA WS. (A) Mobile testing facility in an enclosed trailer for transport. (B) Recreational vehicle retrofitted for DRIT testing. (C) DRIT testing facility in conjunction with university laboratory. Please click here to view a larger version of this figure.

\section{Discussion}

The DRIT is a flexible method suited for field-based surveillance for detecting the presence of rabies virus that can be used in decentralized laboratory areas. While it is possible to conduct the entire test in a field-based setting such as on the tailgate of a truck, it is ideal to have a small, indoor space dedicated for DRIT due to chemical, equipment and supply storage issues. Additionally, adherence to all applicable federal, state, and local laws, and regulations for chemical use and disposal must be considered. Currently, USDA WS has 15 DRIT facilities to test samples from 17 states. The USDA WS DRIT facilities are established in conjunction with university and state public health laboratories, in designated rooms within larger facilities and in enclosed trailers that have been retrofitted and converted to serve as mobile testing units in the event of an emergency outbreak response where enhanced rabies surveillance testing with immediate turnaround time is critical (Figure 5).

While the test has been successful using brainstem material of variable quality, fresh brainstem tissue with no tissue decomposition is optimal. As decomposition, desiccation, or liquefaction occurs, sample quality decreases and the test may detect more nonspecific staining which can 
confuse results. This observation is similar between DFA and DRIT'2. Brainstem/CNS should be collected as soon as possible and then stored frozen $\left(-20^{\circ} \mathrm{C}\right)$ until testing.

Typically, most USDA WS facilities process from 12 to 24 slides at once during a DRIT session, including one positive control and one negative control that have been confirmed via DFA. Positive and negative controls provide a reference point for each DRIT run to ensure the test was successful and to confirm if interpretative questions arise on the test slides. If a sample is not determined to have a clear positive or negative outcome, it is labeled as an indeterminate and tested a second time by DRIT. If this sample is not a clear positive or negative after two DRIT tests, it is sent to a reference laboratory for DFA or related testing.

As with any diagnostic test, 'trouble shooting' is useful with unexpected findings. For example, if a DRIT run is unsuccessful (i.e., the positive control does not exhibit +3 or +4 staining intensity and antigen distribution), ensure all chemicals and reagents have not expired. We have found using a newly opened bottle of hydrogen peroxide at a minimum of once per week is helpful to help prevent non-specific staining through oxidation of the brain tissue. Additionally, we recommend replacing acetate buffer at least once each year at a minimum, regardless of the labeled expiration date.

There are a number of advantages of the DRIT over DFA including lower costs, ability to perform the test outside of a centralized laboratory, need for only light microscopy instead of a fluorescent microscope, and the relatively straightforward training process for people administering and reading the test ${ }^{2,3,4}$. These advantages, coupled with sensitivity and specificity of the DRIT that are comparable to DFA ${ }^{2,7,8,9}$ have already proven that the test serves as an important tool in broad scale enhanced rabies surveillance programs ${ }^{8,10}$ in North America. Additionally, the DRIT has potential to allow for increased surveillance and more prompt testing in developing nations or other areas with limited resources, especially after recent OIE/WHO guidance as a recommended test.

\section{Disclosures}

The authors have nothing to disclose.

\section{Acknowledgments}

We acknowledge all USDA Wildlife Services staff who currently or have previously collected enhanced rabies surveillance samples and have conducted the DRIT for rabies diagnosis. Likewise, we acknowledge the many cooperators who assist us with Enhanced Rabies Surveillance collection. We also thank the Centers for Disease Control and Prevention and The Wistar Institute for access to critical reagents required to conduct the DRIT and for providing training opportunities. In addition, we appreciate the confirmatory diagnostics and technical assistance provided by the Centers for Disease Control and Prevention and by the Wadsworth Center with the New York State Department of Health. Use of any commercial products is for comparison purposes only and does not constitute endorsement.

\section{References}

1. Dean, D. J., Abelseth, M. K., \& Atanasiu, P. in Laboratory techniques in rabies. Vol. 23 eds Meslin F.X., Kaplan M.M., \& Koprowski, H. 88-89 World Health Organization (1996).

2. Durr, S. et al. Rabies diagnosis for developing countries. PLOS Neglected Tropical Diseases. 2 (3), e206, (2008).

3. Rupprecht, C. et al. Progress in the development of a direct rapid immunohistochemical test for diagnosing rabies. (2014).

4. Rupprecht, C. E. et al. Additional Progress in the Development and Application of a Direct, Rapid Immunohistochemical Test for Rabies Diagnosis. Journal of Veterinary Science. 5 (2), (2018).

5. Coleman, P. G., Fevre, E. M., \& Cleaveland, S. Estimating the public health impact of rabies. Emerging Infectious Diseases. 10 (1), $140-142$, (2004).

6. Standard Operating Procedure for the Direct Rapid Immunohistochemistry Test (DRIT) for the detection of rabies virus antigens. Centers for Disease Control and Prevention (2016).

7. Lembo, T. et al. Evaluation of a direct, rapid immunohistochemical test for rabies diagnosis. Emerging Infectious Diseases. 12 (2), $310-313$, (2006).

8. Middel, K., Fehlner-Gardiner, C., Pulham, N., \& Buchanan, T. Incorporating Direct Rapid Immunohistochemical Testing into Large-Scale Wildlife Rabies Surveillance. Tropical Medicine and Infectious Disease. 2 (3), 21, (2017).

9. Coetzer, A., Sabeta, C. T., Markotter, W., Rupprecht, C. E., \& Nel, L. H. Comparison of biotinylated monoclonal and polyclonal antibodies in an evaluation of a direct rapid immunohistochemical test for the routine diagnosis of rabies in southern Africa. PLOS Neglected Tropical Diseases. 8 (9), e3189, (2014).

10. Kirby, J. et al. Enhanced Rabies Surveillance to Support Effective Oral Rabies Vaccination of Raccoons in the Eastern United States. Tropical Medicine and Infectious Disease. 2 (3), 34, (2017).

11. Slate, D. et al. Oral rabies vaccination in north america: opportunities, complexities, and challenges. PLOS Neglected Tropical Diseases. 3 (12), e549, (2009).

12. Direct Rapid Immunohistochemistry Test (DRIT) protocols. <https://rabiessurveillanceblueprint.org/Direct-Rapid-Immunohistochemistry? lang=fr $>$ (2019).

13. Khalid, A., \& Haque, A. Touch Impression Cytology Versus Frozen Section as Intraoperative Consultation Diagnosis. Vol. 2 (2004). 